\title{
An RSSI-based Differential Correlation Algorithm for Wireless Node Localization
}

\author{
Wan $\mathrm{Xu}^{*}$, Zheng Zhang and Guocheng Wan \\ School of Mechanical Engineering, Hubei University of Technology, Wuhan, 430068, Hubei, China
}

\begin{abstract}
The wireless node localization is one of the most significant research issues in the field of wireless sensor networks. This paper presents an RSSI(Received Signal Strength Indicator)-based differential correlation algorithm which sets a correction node near the blind nodes and applies the correction factor and difference coefficient to correct the distance measurement results. In order to verify the performance of the algorithm, a simulation is implemented and the result indicates that the modified algorithm can achieve a absolute error of $1.36 \mathrm{~m}$ and a relative error of $1.91 \%$ in the range of $100 \mathrm{~m} \times 100 \mathrm{~m}$. Compared with the multilateral positioning algorithm, the existence of correction nodes in the algorithm can improve both the absolute accuracy and relative accuracy of the wireless node localization with a better stability.
\end{abstract}

Keywords: Wireless node localization, differential correction algorithm, RSSI.

\section{INTRODUCTION}

Wireless sensor network node localization is to obtain a node's position among the nodes in a self-organizing network through specific localization algorithm [1]. The node localization process can be realized by manually marking or using self-localization algorithm. However, it is difficult to gain the node position manually due to the random and uncontrollable deployment of the nodes. Meanwhile, in some particular fields, the operation of the nodes is in moving status which makes the manual marking impossible. Therefore, the wireless node self-localization technology is very important in the practical applications of WSN.

Numerous of classification methods for wireless sensor node localization algorithms have been reported. One method is based on the distance measured between the nodes, dividing these algorithms into two categories: range-based and range-free localization algorithm.

The principle of range-based localization algorithms is based on the distance measurement between the blind node and the reference nodes, the position of the blind node can then be determined through spatial geometric relationship. The commonly used measurements include TOA, RTOF and TDOA measurement. However, these methods rely on high accuracy on the clock which compromises the application in the wireless sensor networks [2]. PDOA (Phase Difference of Arrival) method calculates the round-trip distance based on the phase differences and round-trip propagation time of the obtained signals The NFER (Near Field EM Ranging) is achieved through measuring of the phase differences between the near-field electromagnetic field. But it suffers from the limitations due to the test distance and electromag netic environment [3, 4]. In RSSL (Received Signal Strength

*Address correspondence to this author at the School of Mechanical Engineering, Hubei University of Technology, Wuhan, 430068, Hubei, China; Tel: +86-27-59750418; Fax+86-27-59750418;

E-mail: xuwan@mail.hbut.edu.cn
Indicator), given the signal propagation loss from the trans mitting node to the receiving node, the distance information can be calculated with the employment of empirical or theoretical signal propagation models [5-7]. Nevertheless, the environment of the wireless sensor increases the complexity transmission losses, which include signal reflection, nonline-of-sight transmission, the multi-path effect, the shadow fading and the antenna direction [8].

The range-free localization technique, on the other hand, is based on the connectivity of the network to determine the number of hops between the nodes as well as the estimation of the distance of each hop based on the information retrieved from the reference nodes [9]. Typically, it consists of the Centroid Localization Algorithm [10], the APIT Localization Algorithm [11] and the DV-hop Localization Algorithm [12]. Yet, due to the difficulty in large-scale network arrangement, these methods are still in the research stage with more simulation applications and less practical uses [13].

This paper proposes a differential correlation algorithm based on RSSI using the multilateral localization principle. Firstly, the correlation nodes are set to be located near the blind nodes. The ranging results are corrected with the correction factor and the difference coefficient. In this way, the systematic errors caused by individual reference node can be eliminated and the localization accuracy can be improved. The paper is organized as follows: sectionIIpresents the RSSI-based differential correlation localization algorithm in details. Section III presents a simulation of the proposed algorithm, and section IV validates the localization system as well as the performance of the algorithm.

\section{RSSI- BASED DIFFERENTIAL CORRELATION LOCALIZATION}

\subsection{Multilateral Localization Principle}

Similar to the triangle localization principle, the multilateral localization principle is to obtain the coordinates of the 


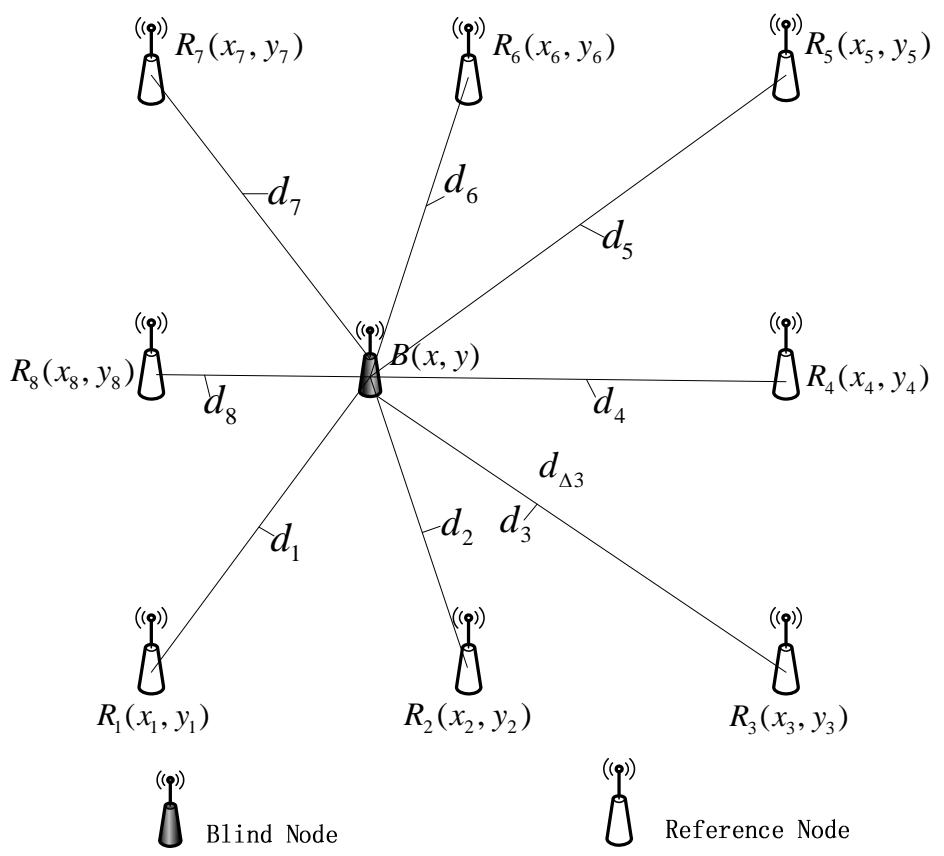

Fig. (1). Multilateral positioning principle.

blind nodes based on the equation established using the reference node coordinates and the distances measured between the reference node and the blind node. In the multilateral localization approach, the employment of three or more reference nodes improves the localization accuracy. The number of distance equations is more than the number of variables to be determined. By applying the maximum likelihood estimation method, the localization becomes more efficient to compensate the measurement error in the case of single reference node, even in the circumstance of communication failure.

As shown in Fig. (1), assuming the number of reference nodes is $\mathrm{n}$, and their coordinates are $\mathrm{R}_{1}\left(\mathrm{x}_{1}, \mathrm{y}_{1}\right), \mathrm{R}_{2}\left(\mathrm{x}_{2}, \mathrm{y}_{2}\right)$, $\mathrm{R}_{3}\left(\mathrm{x}_{3}, \mathrm{y}_{3}\right), \ldots \ldots, \mathrm{R}_{\mathrm{n}}\left(\mathrm{x}_{\mathrm{n}}, \mathrm{y}_{\mathrm{n}}\right)$, the coordinate of blind node is $(\mathrm{x}, \mathrm{y})$, the distances between the reference nodes and blind nodes are $d_{1}, d_{2}, d_{3}, \ldots . ., d n$ respectively.

Assuming

$$
\left\{\begin{array}{c}
\left(x-x_{1}\right)^{2}+\left(y-y_{1}\right)^{2}=d_{1}^{2} \\
\left(x-x_{2}\right)^{2}+\left(y-y_{2}\right)^{2}=d_{2}^{2} \\
\cdots \\
\left(x-x_{n}\right)^{2}+\left(y-y_{n}\right)^{2}=d_{n}^{2}
\end{array}\right.
$$

Expanding Eq. (1), yields,

$$
\left\{\begin{array}{c}
2\left(x_{1}-x_{n}\right) x+2\left(y_{1}-y_{n}\right) y=x_{1}^{2}-x_{n}^{2}+y_{1}^{2}-y_{n}^{2}-d_{1}^{2}+d_{n}^{2} \\
2\left(x_{2}-x_{n}\right) x+2\left(y_{2}-y_{n}\right) y=x_{2}^{2}-x_{n}^{2}+y_{2}^{2}-y_{n}^{2}-d_{2}^{2}+d_{n}^{2} \\
\cdots \\
2\left(x_{n-1}-x_{n}\right) x+2\left(y_{n-1}-y_{n}\right) y=x_{n-1}^{2}-x_{n}^{2}+y_{n-1}^{2}-y_{n}^{2}-d_{n-1}^{2}+d_{n}^{2}
\end{array}\right.
$$

In the format of linear equations,

$$
\left[\begin{array}{cc}
2\left(x_{1}-x_{n}\right) & 2\left(y_{1}-y_{n}\right) \\
2\left(x_{2}-x_{n}\right) & 2\left(y_{2}-y_{n}\right) \\
\cdots & \cdots \\
2\left(x_{2}-x_{n}\right) & 2\left(y_{2}-y_{n}\right)
\end{array}\right]\left[\begin{array}{l}
x \\
y
\end{array}\right]=\left[\begin{array}{c}
x_{1}^{2}-x_{n}^{2}+y_{1}^{2}-y_{n}^{2}-d_{1}^{2}+d_{n}^{2} \\
x_{2}^{2}-x_{n}^{2}+y_{2}^{2}-y_{n}^{2}-d_{2}^{2}+d_{n}^{2} \\
\cdots \\
\cdots \\
x_{n-1}^{2}-x_{n}^{2}+y_{n-1}^{2}-y_{n}^{2}-d_{n-1}^{2}+d_{n}^{2}
\end{array}\right]
$$

The coordinates of the blind nodes can be calculated through the minimum variance estimation,

$$
\hat{X}=\left(A^{T} A\right)^{-1} A^{T} b
$$

\subsection{Differential Correction Localization Algorithm}

In the multilateral localization algorithm, the more reference nodes in unit area, the larger reference node density and the smaller weight of error from individual reference node will be. However, it is in conflict with the requirement of the node density minimization in wireless localization technique. Meanwhile, there is an error accumulation issue in this case. Therefore, the target becomes how to minimize the localization error caused by each reference node in the premise of controlling the node density.

In the premise of limited number of reference nodes, correlation nodes are set near the blind nodes. The ranging results are corrected with the correction factor in relative localization and the difference coefficient. In this way, the system localization accuracy is improved with the reduction of the systematic error caused by individual reference nodes.

The localization principle is illustrated in Fig. (2). Assuming the system consists of one blind node, one correction node and eight Reference nodes. The coordinates of blind node, correction node and reference node are $B(x, y)$, $M(\Delta x, \Delta y)$ and $R_{1}\left(x_{1}, y_{1}\right), R_{2}\left(x_{2}, y_{2}\right), R_{3}\left(x_{3}, y_{3}\right), R_{4}\left(x_{4}, y_{4}\right)$, 


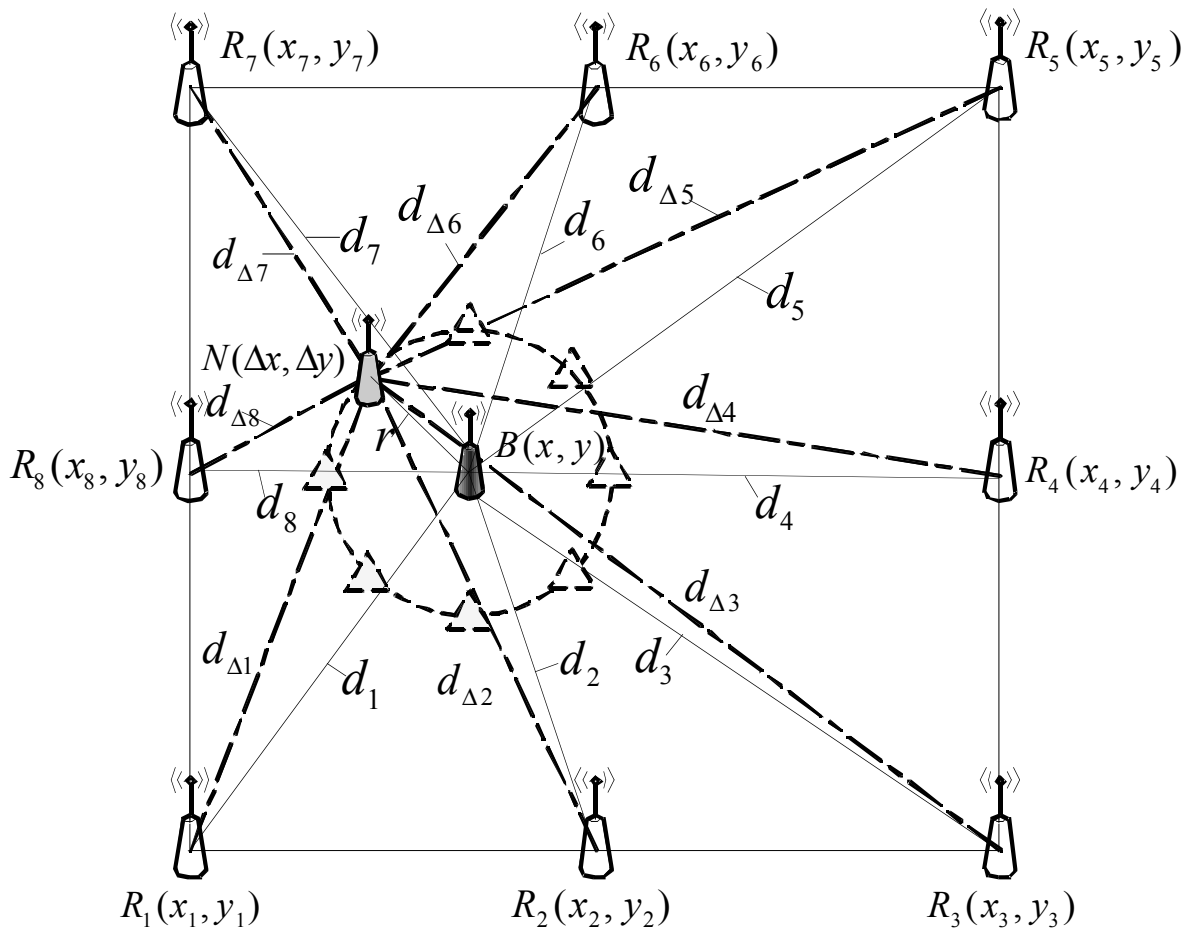

Fig. (2). Differential correction algorithm based on multilateral localization.

$R_{5}\left(x_{5}, y_{5}\right), R_{6}\left(x_{6}, y_{6}\right), R_{7}\left(x_{7}, y_{7}\right), R_{8}\left(x_{8}, y_{8}\right)$. In the network initialization, let the reference nodes be arranged on the circumference with the center at the blind node and the radius equals to $r$.

The definitions of several distance variables are presented in the following:

$$
\begin{aligned}
& d_{\Delta n}: \text { the actual distance between the reference node } \\
& R_{n} \text { and the correction node } \mathrm{M} \text {. }
\end{aligned}
$$

$d_{\Delta n}^{\prime}$ : the measured distance between the reference node $R_{n}$ and the correction node $\mathrm{M}$.

$d_{n}^{\prime}$ : the measured distance between the reference node $R_{n}$ and the blind node $\mathrm{B}$.

$d_{n}$ : the modified distance between the reference node $R_{n}$ and the blind node B after the correction algorithm.

The correction factor $\eta$, which indicates the sum of relative measurement errors between the reference nodes and the correction node, is given as follows:

$$
\begin{aligned}
\eta= & \left(\frac{d_{\Delta 1}^{\prime}-d_{\Delta 1}}{d_{\Delta 1}^{\prime}}\right)+\left(\frac{d_{\Delta 2}^{\prime}-d_{\Delta 2}}{d_{\Delta 2}^{\prime}}\right)+\left(\frac{d_{\Delta 3}^{\prime}-d_{\Delta 3}}{d_{\Delta 3}^{\prime}}\right)+\left(\frac{d_{\Delta 4}^{\prime}-d_{\Delta 4}}{d_{\Delta 4}^{\prime}}\right) \\
& \left(\frac{d_{\Delta 5}^{\prime}-d_{\Delta 5}}{d_{\Delta 5}^{\prime}}\right)+\left(\frac{d_{\Delta 6}^{\prime}-d_{\Delta 6}}{d_{\Delta 6}^{\prime}}\right)+\left(\frac{d_{\Delta 7}^{\prime}-d_{\Delta 7}}{d_{\Delta 7}^{\prime}}\right)+\left(\frac{d_{\Delta 8}^{\prime}-d_{\Delta 8}}{d_{\Delta 8}^{\prime}}\right) \\
= & \left(1-\frac{d_{\Delta 1}^{\prime}}{d_{\Delta 1}^{\prime}}\right)+\left(1-\frac{d_{\Delta 2}}{d_{\Delta 2}^{\prime}}\right)+\left(1-\frac{d_{\Delta 3}}{d_{\Delta 3}^{\prime}}\right)+\left(1-\frac{d_{\Delta 4}}{d_{\Delta 4}^{\prime}}\right) \\
& \left(1-\frac{d_{\Delta 5}}{d_{\Delta 5}^{\prime}}\right)+\left(1-\frac{d_{\Delta 6}}{d_{\Delta 6}^{\prime}}\right)+\left(1-\frac{d_{\Delta 7}}{d_{\Delta 7}^{\prime}}\right)+\left(1-\frac{d_{\Delta 8}}{d_{\Delta 8}^{\prime}}\right) \\
= & 8-\sum_{n=1}^{8} \frac{d_{\Delta n}}{d_{\Delta n}^{\prime}}
\end{aligned}
$$

The variation coefficient from the reference node $R_{n}$ to the blind node is defined as

$\mu_{n}=\lambda e^{1-\frac{d_{n}^{\prime}}{d_{\Delta n}^{\prime}(1-\eta)}}$

In Eq. (6), $\lambda$ is the adjustable factor ranging from 0 to 1 , which is obtained from the actual experimental environment at the beginning of the arrangement.

The distance error between the reference node $R_{n}$ and the correction node is defined as

$\varepsilon_{n}=d_{\Delta n}^{\prime}-d_{\Delta n}$

Thus, the modified distance can be obtained as

$d_{n}=d_{n}^{\prime}-\mu_{n} \varepsilon_{n}$

Finally, the blind node coordinate $(x, y)$ can be determined with Eq. (4).

\section{ALGORITHM SIMULATION}

In order to verify the performance of the algorithm, this paper proposes a simulation test with Matlab. In this test, a few assumptions have been made. First, assuming the test area is $100 \mathrm{~m} \times 100 \mathrm{~m}$ with eight reference nodes arranged in a symmetrical manner in this area. Their coordinates are $(10$, $10),(40,10),(70,10),(70,40),(70,70),(40,70),(10,70)$, $(10,40)$. Secondly, let the correction node be arranged on the circumference with center at the blind node and its radius equals to $r$, the angle between the correction node and blind node is $\alpha$.

In the simulation process, in order to analyze the influence from different position arrangements to the correction accuracy, four sets of data are measured: (a) $r=10 \mathrm{~m}$, 
$\alpha=0^{\circ}$; (b) $r=10 \mathrm{~m}, \alpha=180^{\circ}$; (c) $r=20 \mathrm{~m}, \alpha=0^{\circ}$;

(d) $r=20 m, \quad \alpha=180^{\circ}$.

By comparing the Matlab simulation results shown in Fig. (3), it is identified that the location of the correction nodes has influence on the localization accuracy. The impact is illustrated mainly in two aspects: (1) the smaller distance between the correction node and blind node, the higher localization accuracy; (2) the position errors of blind node and the correction node have the same direction.

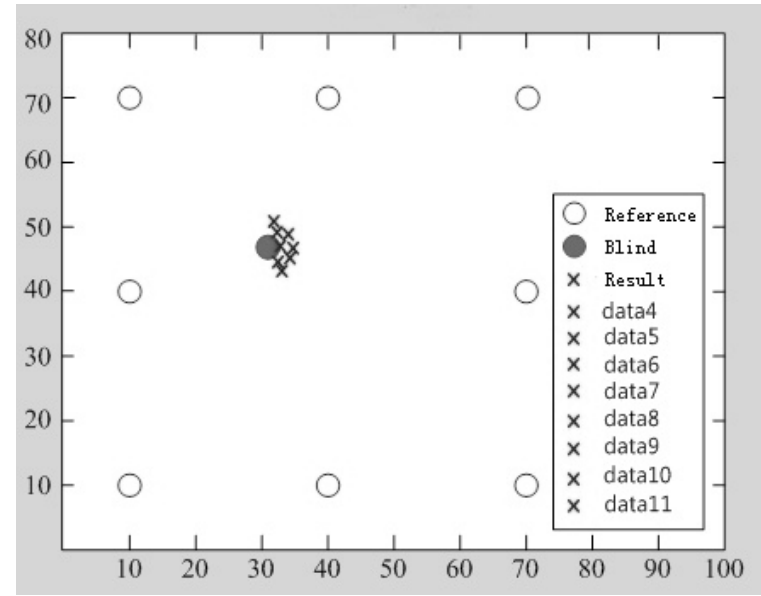

(a) $r=10 m, \alpha=0^{\circ}$

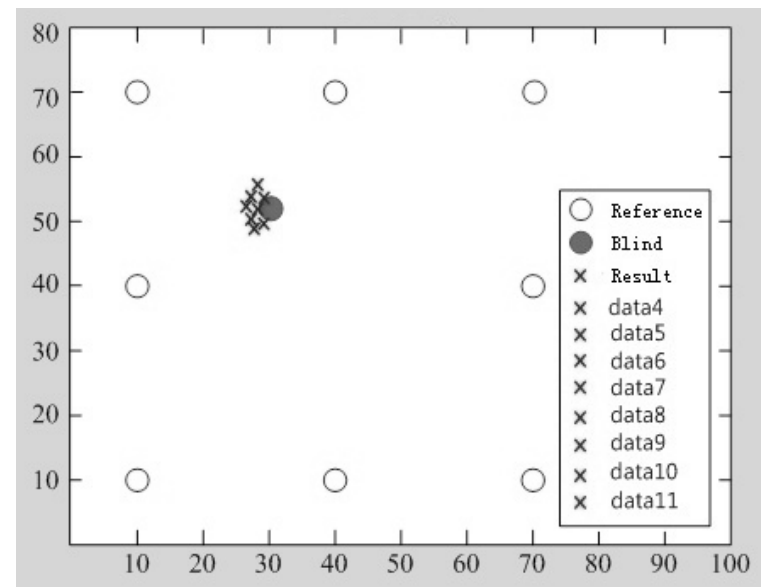

(b) $r=10 m, \alpha=180^{\circ}$

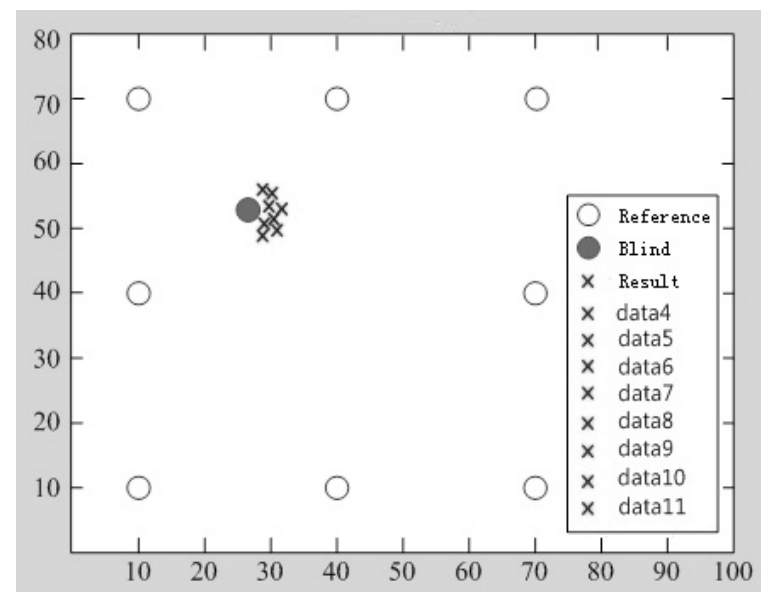

(c) $r=20 m, \alpha=0^{\circ}$

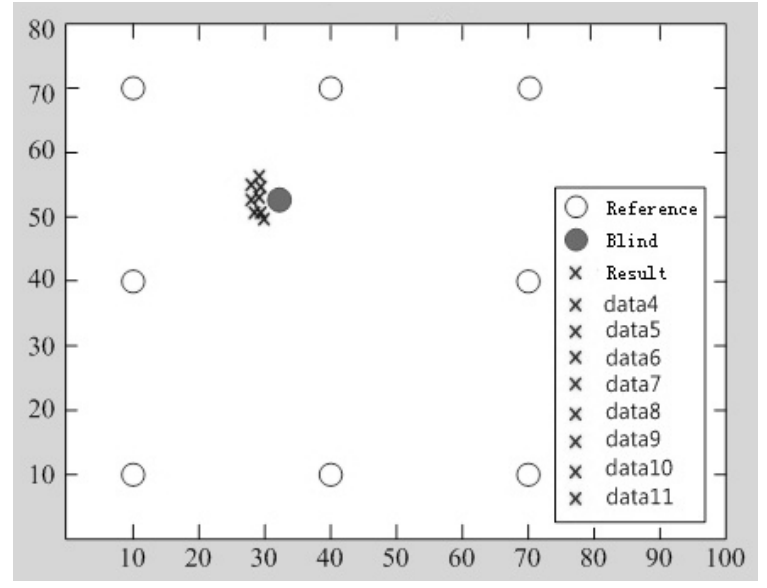

(d) $r=20 m, \alpha=180^{\circ}$

Fig. (3). Simulation results of differential correction algorithm.

To quantify the performance of the localization algorithm, an error analysis is given with the above case $b$ ( $r=10 m, \quad \alpha=180^{\circ}$ ) as an example.

Absolute Error of individual node is defined as

$E_{a}^{i}=\left\|r_{\text {est }}^{i}-r_{\text {real }}^{i}\right\|$

Relative Error of individual node is defined as

$E_{r}^{i}=\frac{E_{a}^{i}}{R} \times 100 \%$

In Eqs. (9) and (10), the measurement position and actual position are denoted as $r_{\text {est }}^{i}$ and $r_{\text {real }}^{i}$, and $\mathrm{R}$ is the maximum communication distance ( $\mathrm{R}$ is taken $70 \mathrm{~m}$ ). In the area of $100 \mathrm{~m} \times 100 \mathrm{~m}$, changing the location of blind node, and substituting the calculated data from Matlab to Eqs. (9) and (10), the results can be obtained in Table 1 .

The simulation result indicates that the modified algorithm can achieve an absolute error of $1.36 \mathrm{~m}$ and a relative error of $1.91 \%$ in the range of $100 \mathrm{~m} \times 100 \mathrm{~m}$.

\section{EXPERIMENT}

This paper establishes an area $(30 \mathrm{~m} \times 20 \mathrm{~m})$ as a wireless localization system which includes six CC2430 as Reference Nodes, one CC2431 as Blind Node and two CC2439 as coordinate node and correction node. The localization information of the blind node is calculated through the RSSI-based differential correction algorithm, which is sent to the coordinate node periodically. Finally, the data is uploaded to the host computer through RS232 from the coordinate node.

The overall structure of the system is shows in Fig. (4).

There are two groups of tests to evaluate the localization accuracy: One group concentrates on the modified algorithm with a correction node. The other group focuses on the multilateral localization algorithm without correction node.

The coordinates of the six reference nodes are $(2,2),(17$, $2),(32,2),(32,22),(17,22),(2,22)$ respectively, and the angle between the correction node and blind node is defined 
Table 1. Algorithm Error Analysis

\begin{tabular}{|c|c|c|c|c|}
\hline No. & $\boldsymbol{r}_{\text {est }}$ & $\boldsymbol{r}_{\text {real }}$ & $E_{a}$ & $E_{r}(\%)$ \\
\hline \hline 1 & $(20,20)$ & $(18.8,21.1)$ & 1.62 & 2.32 \\
\hline 2 & $(40,20)$ & $(38.9,20.9)$ & 1.42 & 2.03 \\
\hline 3 & $(60,20)$ & $(58.3,20.5)$ & 1.58 & 1.08 \\
\hline 4 & $(20,40)$ & $(19.1,39.4)$ & 0.76 & 1.54 \\
\hline 5 & $(40,40)$ & $(39.3,40.3)$ & 1.34 & 1.08 \\
\hline 6 & $(60,40)$ & $(58.8,39.4)$ & 1.36 & 1.92 \\
\hline 7 & $(20,60)$ & $(19.2,58.9)$ & 1.34 & 1.94 \\
\hline 8 & $(40,60)$ & $(39,59.1)$ & 1.70 & 2.43 \\
\hline 9 & $(60,60)$ & $(58.9,58.7)$ & & $E_{r}=1.91$ \\
\hline
\end{tabular}

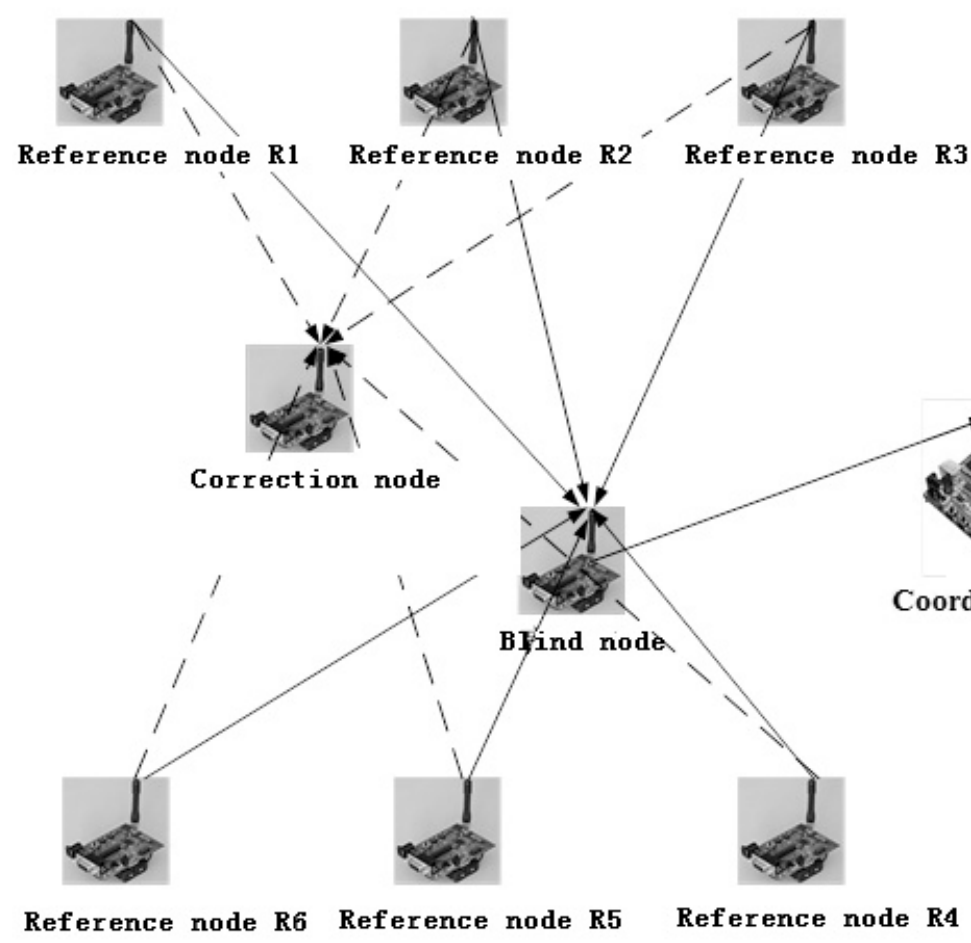

Fig. (4). Overall architecture of the system.

as $\alpha=0^{\circ}$ with the distance of $r=12 \mathrm{~m}$, the adjustable factor is designed as $\lambda=0.6$. As soon as the nodes are initialized, the localization information of the blind nodes calculated with the modified RSSI-based differential correction algorithm is sent to the coordinate node in the period of $100 \mathrm{~ms}$. Finally, the data is uploaded to the host computer through RS232 from the coordinate node as shown in Fig. (5).

In the testing process, the six reference nodes are fixed, the blind node and the correction node are movable in the testing area. In addition, the blind nodes are evenly arranged in the middle of test area with a square area of $16 \mathrm{~m} \times 16 \mathrm{~m}$. The results are shown in Table 2 . From Table 2 , it can be concluded that, compared with the multilateral localization algorithm without correction nodes, the existence of correction nodes with the modified algorithm benefits the absolute accuracy and the relative accuracy. Meanwhile, the variance of the modified algorithm is 0.22 , which indicates the system has a better stability.

\section{CONCLUSION}

This paper proposes a modified RSSI-based differential correlation algorithm. In the premise of the limited number of reference nodes, correction nodes are set near the blind nodes. The ranging results are corrected with the correction factor in relative positioning and the difference coefficient. In this way, the system accuracy is improved with the reduc- 


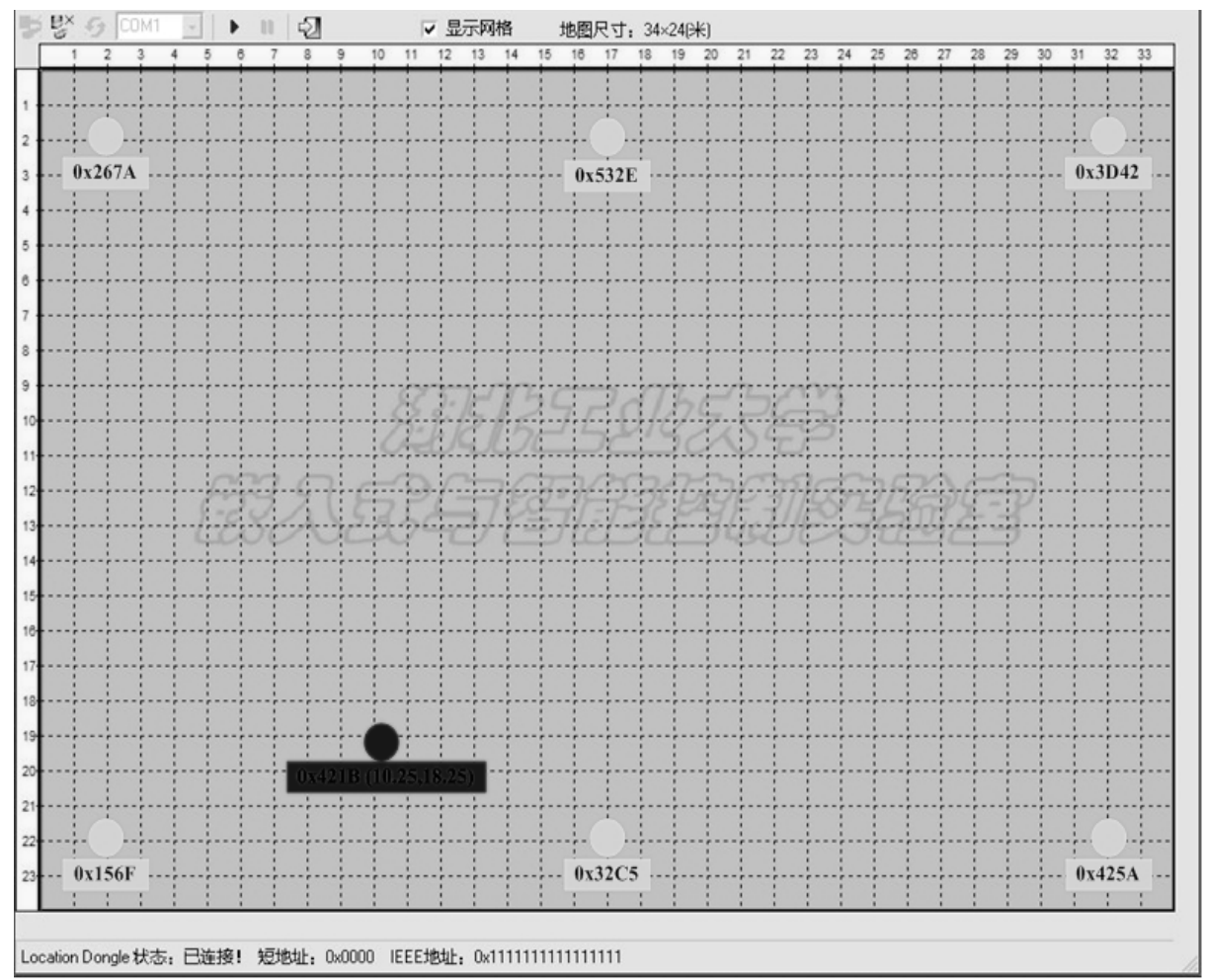

Fig. (5). Localization monitoring interface.

Table 2. Test Results

\begin{tabular}{|c|c|c|c|c|c|c|c|}
\hline \multirow[b]{2}{*}{ No. } & \multirow[b]{2}{*}{ Actual Position } & \multicolumn{3}{|c|}{ Without Correction Node } & \multicolumn{3}{|c|}{ With Correction Node } \\
\hline & & Measured Value & $\begin{array}{l}\text { Absolute } \\
\text { Error } \\
E_{a}(\mathbf{m})\end{array}$ & $\begin{array}{c}\text { Relative Error } \\
\qquad E_{r}(\%)\end{array}$ & Measured Value & $\begin{array}{l}\text { Absolute } \\
\text { Error } \\
E_{a}^{\prime}(\mathbf{m})\end{array}$ & $\begin{array}{c}\text { Relative } \\
\text { Error } \\
E_{r}^{\prime}(\%)\end{array}$ \\
\hline 1 & $(9,4)$ & $(11,2.75)$ & 2.35 & 4.7 & $(9.75,3.25)$ & 1.06 & 2.1 \\
\hline 2 & $(17,4)$ & $(18.75,3)$ & 2.02 & 4.0 & $(17.5,3.25)$ & 0.90 & 1.8 \\
\hline 3 & $(25,4)$ & $(23.25,2.75)$ & 2.15 & 4.3 & $(24.5,3)$ & 1.12 & 2.2 \\
\hline 4 & $(9,12)$ & $(10.5,11)$ & 1.80 & 3.6 & $(9.75,11.25)$ & 1.06 & 2.1 \\
\hline 5 & $(17,12)$ & $(16.25,11)$ & 1.25 & 2.5 & $(17.5,12.25)$ & 0.56 & 1.1 \\
\hline 6 & $(25,12)$ & $(26,10.75)$ & 1.60 & 3.2 & $(24.75,11.25)$ & 0.79 & 1.6 \\
\hline 7 & $(9,20)$ & $(10.75,18.5)$ & 2.30 & 4.6 & $(9.5,19.5)$ & 0.71 & 1.4 \\
\hline 8 & $(17,20)$ & $(17.75,18.25)$ & 1.90 & 3.8 & $(16.75,19)$ & 1.03 & 2.1 \\
\hline 9 & $(25,20)$ & $(26,17.75)$ & 2.46 & 4.9 & $(25.25,18.75)$ & 1.27 & 2.5 \\
\hline \multicolumn{2}{|c|}{ Total/Summary } & \multicolumn{3}{|c|}{$\begin{array}{c}E_{a}=1.98, \quad E_{a}=3.96 \\
- \\
S\left(E_{a}\right)=0.39\end{array}$} & \multicolumn{3}{|c|}{$\begin{array}{c}E_{a}^{\prime}=0.94, \quad E_{r}^{\prime}=1.87 \\
\overline{-} \\
S\left(\overline{E_{a}^{\prime}}\right)=0.22\end{array}$} \\
\hline
\end{tabular}

tion of the systematic error caused by individual reference nodes. The simulation results indicate that the modified algorithm can achieve an absolute error of $1.36 \mathrm{~m}$ and a relative error of $1.91 \%$ in the testing area of $100 \mathrm{~m} \times 100 \mathrm{~m}$. By experimental comparisons with the multilateral positioning algorithm without modifying nodes, the existence of correction nodes with the proposed algorithm enhances not only the absolute accuracy and relative accuracy, but also the stability of the modified system.

\section{CONFLICT OF INTEREST}

The authors confirm that this article content has no conflicts of interest. 


\section{ACKNOWLEDGEMENTS}

This work was supported by Educational Commission of Hubei Province of China Grant No. Q20111403, and the Youth Chenguang Project of Science and Technology of Wuhan City of China, Grant No. 201271031386.

\section{REFERENCES}

[1] S. S. Wang, M. Green and M. Malkawa, "E-911 Location Standards and Location Commercial Services", Proceeding of IEEE Emerging Technologies Symposium on Broadband, Wireless Internet Access, pp.1-5, 2000.

[2] M. Wiebking, J. Hoffmann, C. Heide, "Wireless local positioning", IEEE Microwave Magazine, vol. 4, no. 4, pp. 77-86, 2003.

[3] N. Bulusu, J. Heidemann, D. Estrin, "Density adaptive algorithms for beacon placement in wireless sensor networks", Technical Report ULCA-CS-010013, 2001.

[4] D. Moore, J. Leonard and S. Teller, "Robust distributed network localization with noisy range measurement", Proceedings of the 2nd international conference on Embedded networked sensor systems, pp. 50-61, 2004.

[5] F. Ren, H. Huang and C. Lin, "Wireless sensor network", Journal of Software, vol. 14, no.7, pp. 282-291, 2003.
[6] X. Zhang, Y. Wang and Q. Guo, "Research on the indoor channel fading model in WSN", Journal of Shandong University, vol. 43, no. 11 , pp. 44-47, 2008.

[7] J. F Akyildiz, W. Su, "Wireless sensor networks:a survey", Computer Networks, vol. 38, no. 4, pp. 393-422, 2002.

[8] Z. Fang, S. Zhao and P. Guo, "Analysis of Distance Measurement Based on RSSI”, Chinese Journal of Sensors and Actuators, vol.20, no. 11, pp. 2526-2530, 2007.

[9] L. Sun, J. Li and Y. Chen, Wireless sensor network Beijing, Tsinghua University Press, Beijing 2005.

[10] X. Shen, Z. Wang, P. Jiang, R. lin and Y. Sun, "Connectivity and RSSI Based Localization Scheme for Wireless Sensor Networks", Lecture Notes on Computer Science, vol. 3645, pp.578-587, 2005.

[11] T. He, C. Huang, B.M. Blum, J.A. Stankovic and T. Abdezaher, "Range-Free Localization Schemes for Large Scale Sensor Networks", Proceedings of the 9th annual international conference on Mobile computing and networking, pp.81-95, 2003.

[12] P. C. Chen, "A non-line-of-sight error mitigation algorithm", Proceedings of IEEE Wireless Communications and Networking Conference, pp. 316-320, 1999.

[13] X. Jiang, "Study on range- free based on self-location algorithms in wireless Sensor networks", Master Thesis, Southwest Jiaotong University, China, 2007.

(C) Xu et al.; Licensee Bentham Open.

This is an open access article licensed under the terms of the Creative Commons Attribution Non-Commercial License (http://creativecommons.org/licenses/by-nc/3.0/) which permits unrestricted, non-commercial use, distribution and reproduction in any medium, provided the work is properly cited. 